\title{
Alteração da carga de ectoparasitas em vacas da raça Holandesa submetidas a diferentes níveis de alho (Allium sativum L.) na alimentação
}

\author{
MASSARIOL, P.B. ${ }^{1}$; OLIVO, C.J. ${ }^{1 *}$; RICHARDS, N. ${ }^{2}$; AGNOLIN, C.A. ${ }^{1}$; MEINERZ, G.R. ${ }^{1}$; BOTH, J.F. ${ }^{4}$; FACCIO, \\ L. ; HOHENREUTHER, F. ; MARTINELLI, S. ${ }^{1}$ \\ Universidade Federal de Santa Maria (UFSM), Centro de Ciências Rurais, Departamento de Zootecnia, Santa \\ Maria-RS, Brasil, 97105-900, ${ }^{*}$ clairo@ccr.usfm.br. ${ }^{2}$ Universidade Federal de Santa Maria (UFSM), Centro de \\ Ciências Rurais, Departamento de Tecnologia e Ciência dos Alimentos, UFSM, RS, Brasil.
}

\begin{abstract}
RESUMO: Dezesseis vacas de raça Holandesa em lactação foram utilizadas para avaliar o efeito da administração de alho na alimentação sobre a alteração da carga de carrapato, mosca-dochifre, mosca dos estábulos e mosca doméstica. Os tratamentos $(\mathrm{T})$ foram constituídos por controle negativo (T1), alho a $100 \mathrm{~g}$ (T2) e $200 \mathrm{~g} \mathrm{vaca}^{-1}$ (T3), por três dias, e o amitraz a 0,025\% (T4). Para avaliação, foram contadas as fêmeas ingurgitadas de carrapato com comprimento superior a quatro mm e as moscas, antes da aplicação e no 1, 2, 3, 4, 5, 6, 7 e 14ํํㅁ dia após a aplicação de cada tratamento. Nos 1, 2 e 3 o dias pós-tratamento foram coletadas amostras de leite para avaliação das propriedades organolépticas do leite cru e do queijo Minas-Frescal. Os valores médios de carrapatos, em comparação ao pré-tratamento, foram de 188,$44 ; 106,28$; 108,10 e $7,60 \%$, respectivamente. O ectoparasiticida comercial foi o mais eficaz $(p<0,05)$ no controle de carrapatos. Os animais que receberam alho (T2) tiveram redução da contagem de carrapatos, no 14ㅇdia, sendo (T2 e T3) similares $(p>0,05)$ ao produto químico (T4). Nestes tratamentos, não foi detectado odor ou sabor de alho no leite e no queijo. Não houve diferença $(p>0,05)$ entre os tratamentos constituídos no controle de moscas.
\end{abstract}

Palavras-chave: Allium sativum, amitraz, Boophilus microplus, Haematobia irritans, Stomoxys calcitrans

\begin{abstract}
Ectoparasite load alteration in Holstein cows fed with different garlic (Allium sativum L.) levels. Sixteen lactating Holstein cows were used to evaluate the effect of garlic supply on the load of tick, horn fly, stable fly and house fly. Treatments (T) consisted of a negative control (T1), garlic at $100 \mathrm{~g} \mathrm{(T2)} \mathrm{and} 200 \mathrm{~g} \mathrm{cow}^{-1}$ (T3) for three days, and Amitraz $0.025 \%$ (T4). Engorged female ticks presenting length superior to $4.0 \mathrm{~mm}$ and flies were counted before application and on the $1^{\text {st }}, 2^{\text {nd }}, 3^{\text {rd }}, 4^{\text {th }}, 5^{\text {th }}, 6^{\text {th }}, 7^{\text {th }}$, and $14^{\text {th }}$ day after each treatment application. On the $1^{\text {st }}, 2^{\text {nd }}$, $3^{\text {rd }}$ days after treatments, milk samples were collected to evaluate organoleptic properties in raw milk and in "Minas-Frescal" cheese. Tick mean values were 188.44, 106.28, 108.10, and 7.60\%, respectively, relative to those of the pretreatment. Commercial ectoparasiticide was the most efficient $(p<0.05)$ for tick control. On the $14^{\text {th }}$ day, garlic-treated animals $(T 2)$ had a reduced tick number, and T2 and T3 had a tick number similar ( $p>0.05$ ) to that of the chemical product (T4). In these treatments, no garlic flavor or taste was detected in milk and cheese. There was no difference $(p>0.05)$ among treatments for fly control.
\end{abstract}

Key words: Allium sativum, amitraz, Boophilus microplus, Haematobia irritans, Stomoxys calcitrans

\section{INTRODUÇÃO}

O parasitismo é um dos fatores que causa diminuição da eficiência produtiva dos animais (Bianchin et al., 1999), implicando em grandes perdas econômicas. Os prejuízos causados por parasitas externos em rebanhos bovinos no Brasil superam a cifra de dois bilhões de dólares ao ano, dos quais

Recebido para publicação em 13/11/2007

Aceito para publicação em 10/06/2008

Rev. Bras. PI. Med., Botucatu, v.11, n.1, p.37-42, 2009. 
$75 \%$ são atribuídos ao carrapato e $25 \%$ às demais parasitoses (especialmente mosca-dos-chifres, berne, miíases, mosca dos estábulos) que somadas correspondem aos $25 \%$ restantes (Grisi et al., 2002).

Para o controle convencional de parasitas, tanto de animais quanto de plantas, normalmente são utilizados pesticidas, responsáveis por taxas consideráveis de intoxicações e óbitos da população humana, especialmente de trabalhadores rurais (Faria et al., 2007). Somado a esta problemática, tem-se ainda a contaminação por produtos antiparasitários no leite e na carne disponíveis para alimentação humana, além da contaminação ambiental direta (Molento et al., 2004). Agregam-se ainda problemas com o desenvolvimento da resistência aos produtos químicos utilizados (Furlong, 2004), especialmente em rebanhos de bovinos leiteiros (Oliveira \& Azevedo, 2002). A indústria, por sua vez, tem hesitado em investir na pesquisa de novos produtos químicos, não só pelos custos envolvidos que são maiores em relação aos sintéticos em décadas passadas (Jonsson, 2006), além do tempo de comercialização de um novo produto que é de difícil cálculo, certamente limitado em função da aquisição de resistência dos parasitas (Chagas et al., 2003).

Neste contexto, em diferentes regiões do mundo, têm-se buscado alternativas visando diminuir o uso de parasiticidas sintéticos, havendo destaque para os produtos fitoterápicos. Sua utilização pode reduzir os impactos ambientais e econômicos causados pelo uso de produtos sintéticos convencionais, ressaltando-se que o desenvolvimento da resistência dos artrópodes aos fitoterápicos (compostos por associações de vários princípios ativos) é um processo geralmente lento (Roel, 2002; Chungsamarnyart \& Jiwajinda, 1992). Soma-se a isso, também, a expansão da agricultura orgânica, implicando necessariamente, em se ter alternativas mais eficientes no controle de parasitas, considerando-se que esta estratégia de produção não permite o uso de pesticidas. Ainda, o uso de fitoterápicos em sistemas agrícolas convencionais, como parte da estratégia de controle de parasitoses, pode estender a vida útil dos produtos químicos (Vieira \& Cavalcante, 1999).

Os agricultores de maneira geral têm facilidade de acesso e de obtenção dos fitoterápicos, além destes, normalmente, não deixarem resíduos na alimentação e apresentarem baixo custo de produção (Roel, 2002). No entanto, além de existirem poucas pesquisas sobre o uso de fitoterápicos no controle de parasitas de bovinos, as diferenças, quanto às características de ambiente e forma de cultivo, colheita e conservação dos produtos, podem implicar na oscilação dos resultados (Heimerdinger et al., 2006).

Dentre os fitoterápicos, destaca-se o alho, que além do uso generalizado como condimento, são atribuídas a ele qualidades terapêuticas. O alho é rico em substâncias organossulfuradas e dentre estas se destacam a alicina (Ankri \& Mirelman, 1999) e o ajoeno (Urbina et al., 1993), principais responsáveis pelos efeitos antiparasitários. Devido a composição química, no entanto, as plantas e subprodutos do gênero Allium podem transferir substâncias típicas do sabor do alho para o leite, interferindo nas propriedades organolépticas dos produtos lácteos (Glasier, 1960).

O uso como ectoparasiticida, embora indicado pelo conhecimento popular, cientificamente tem sido pouco estudado. Assim, o objetivo deste trabalho foi avaliar os efeitos do alho administrado à alimentação, no controle de ectoparasitas de bovinos e nas características organolépticas do leite e queijo.

\section{MATERIAL E MÉTODO}

O experimento foi realizado no Laboratório de Bovinocultura de Leite (DZ, UFSM), de dezembro de 2006 a janeiro de 2007. Foram testados quatro tratamentos, constituídos por controle negativo ( $\mathrm{T} 1$ ), alho picado e misturado ao concentrado a $100 \mathrm{~g}$ (T2) e $200 \mathrm{~g} \mathrm{vaca}^{-1} \mathrm{dia}^{-1}$ (T3), e por amitraz a 0,025\% (T4). Para o T2 e o T3, o fornecimento foi repetido no $2^{\circ} \mathrm{e}$ 3 ㅇ dias.

Como unidades experimentais foram usadas 16 vacas da raça Holandês (preto e branco), quatro por tratamento. Os animais possuíam cerca de 500 $\mathrm{kg}$ de peso vivo e produção de leite entre 17 e $18 \mathrm{~kg}$ de leite por dia, sendo manejadas em conjunto e naturalmente infestadas por carrapatos. As vacas foram submetidas diariamente a duas ordenhas e, entre elas, manejadas em áreas constituídas por pastagens tropicais, recebendo como complemento alimentar $3 \mathrm{~kg}$ de concentrado $\mathrm{dia}^{-1}$.

Como parâmetro de controle do carrapato, consideraram-se os ínstares com tamanho igual ou superior a $4 \mathrm{~mm}$ de comprimento. Os animais foram distribuídos em cada tratamento quando apresentavam em torno de 20 teleóginas. Uma vez selecionados, foram feitas contagens de moscas-dos-chifres e da mosca doméstica (+) mosca dos estábulos. Os animais sorteados para o grupo controle positivo foram banhados individualmente com quatro litros da solução constituída por água e Amitraz, usando-se um pulverizador costal. Nos tratamentos constituídos por alho, os bulbilhos destinados a cada animal foram grosseiramente cortados, agregando-se $50 \mathrm{~g}$ de açúcar mascavo diluído em água aquecida, e misturados ao concentrado, fornecido após a ordenha da tarde. Essa forma de administração e as quantidades foram baseadas nos estudos conduzidos por Bianchin et al. (1999), Alvarenga et al. (2004) e por testes pré-experimentais

Rev. Bras. Pl. Med., Botucatu, v.11, n.1, p.37-42, 2009. 
efetuados com animais do rebanho do Laboratório de Bovinocultura de Leite (DZ-UFSM). Dentre as formas de administração testadas optou-se pelo uso do produto in natura, sendo grosseiramente desintegrado (três a quatro partes por bulbilho), favorecendo a ingestão do produto com o concentrado. Diferentemente das pesquisas conduzidas com alho que fornecem pequenas quantidades por determinado tempo, optou-se por usar quantidades elevadas em curto período, comparado com o procedimento seguido por Alvarenga et al. (2004), visando observar efeitos (dessa forma de administração) sobre os ectoparasitas e, especialmente, sobre as propriedades organolépticas do leite.

Após a aplicação dos tratamentos foram efetuadas contagens diárias dos ectoparasitas até o $7^{\circ}$ e $14^{\circ}$ dias. Os dados foram transformados em valores percentuais e em logaritmo na base 10 para diminuir a variabilidade.

Para a análise das características sensoriais do leite, as amostras dos 2, 3 e 4음 dias, após a aplicação dos tratamentos, foram avaliadas no laboratório pertencente ao Departamento de Ciência e Tecnologia dos Alimentos (UFSM). Para o produto químico respeitou-se o período de carência indicado pelo fabricante, usando-se as amostras do 3 e $4^{\circ}$ dias. Para análise da cor, utilizou-se o teste de escala hedônica não estruturado de $9 \mathrm{~cm}$ entre âncoras, atribuindo-se posteriormente valores relativos entre "muito claro (estranho)", "normal (bom)" e "muito forte". Para a avaliação do odor utilizou-se a escala hedônica do mesmo tamanho, com valores entre: "muito fraco", "bom" e "muito forte (estranho)". As avaliações foram feitas por cinco provadores experientes (Dutcosky, 1996).

Para análise sensorial do queijo (tipo Minas-Frescal), quanto a cor, aroma, sabor e textura, também se utilizou escalas hedônicas de $9 \mathrm{~cm}$ entre âncoras variando do "desgostei muito" até "gostei muito". Para o atributo sabor residual, as âncoras utilizadas foram "muitíssimo fraco", "normal" e "muitíssimo forte". Para a aparência global foram atribuídas notas de 1 (ruim) a 9 (muito bom) (Lawless \& Heymann, 1998). As análises do queijo foram feitas por doze provadores.

O delineamento experimental utilizado foi inteiramente ao acaso com quatro repetições (vacas). Os resultados foram submetidos à análise de variância ao nível de $5 \%$ de probabilidade do erro (SAS, 1997).

\section{RESULTADO E DISCUSSÃO}

Os valores da análise laboratorial do alho utilizado no experimento, quanto aos teores de matéria seca e proteína bruta, foram de 33,2 e $19,16 \%$, respectivamente. Análises químicas do alho feitas por Alvarenga et al. (2004) revelaram valores similares ao produto usado no presente trabalho quanto à proteína bruta, entre 19,97 e $20 \%$.

Os resultados referentes ao efeito do alho no controle de ectoparasitas encontram-se na Tabela 1. Para o controle de carrapatos, observou-se que houve diferença significativa $(p<0,05)$ entre os tratamentos, tanto entre os dias de avaliação quanto entre as médias. No grupo de animais testemunha, verificou-se um aumento da infestação em cerca de $238 \%$ em 14 dias de avaliação. No grupo de animais que receberam amitraz (controle positivo), a eficácia de controle foi superior a 95\% na primeira semana. $\mathrm{Na}$ segunda semana observou-se um aumento da infestação, confirmando a menor ação do produto sobre as formas jovens do carrapato (Heimerdinger et al., 2006).

Para os tratamentos constituídos por diferentes níveis de alho, não houve diferença significativa $(p<0,05)$ entre eles, tanto nos dias de avaliação quanto entre as médias (Tabela 1). Observase que nos animais tratados com fitoterápicos não houve grandes alterações na carga parasitária média, que se manteve próxima aos valores de prétratamento, mostrando comportamento intermediário $(p<0,05)$ entre os tratamentos constituídos pelo produto químico e o grupo testemunha. Estudos conduzidos com alho no controle do carrapato, além de escassos, demonstram resultados conflitantes. Bianchin et al. (1999), avaliando o uso de alho em pó a $2 \%$ no sal mineral para bovinos mestiços $\left(8 \mathrm{mg} \mathrm{kg}^{-1}\right.$ de peso vivo dia $^{-1}$ ), não observaram efeito do produto no controle de carrapatos. Já no trabalho conduzido por Alvarenga et al. (2004), com resíduo de beneficiamento de alho em bovinos leiteiros mestiços, usando níveis de 0, 3, 6, e $9 \mathrm{~g}_{\text {animal }}{ }^{-1} \mathrm{dia}^{-1}$, comparado com um produto comercial (doramectin), aplicado mensalmente, houve redução da carga de carrapatos, com similaridade de controle até o 56 dia para os níveis com 3 e 6 g e além do 70 dia para o nível de 9 $\mathrm{g}$ em relação ao ectoparasiticida comercial.

Os estudos conduzidos por Alvarenga et al. (2004), visando estudar a ação parasiticida do alho, apontam para a dificuldade de entendimento dos mecanismos de ação de seus componentes sobre 0 carrapato. Pesquisa efetuada com extrato de alho envelhecido demonstra que houve um forte aumento na atuação de células e componentes imunológicos (interleucina 2, interleucina 12 e fator de necrose tumoral alfa) em ratos (Kyo et al., 2001). Esses efeitos poderiam elevar a ação do sistema imunológico dos bovinos, agindo contra as moléculas ligadas à fixação das larvas de carrapato, dificultando a adesão e impedindo o desenvolvimento delas na pele (Andreotti, 2004).

Os resultados obtidos na presente pesquisa confirmam a ação do alho sobre carrapatos, embora

Rev. Bras. PI. Med., Botucatu, v.11, n.1, p.37-42, 2009. 
TABELA 1. Porcentagem de ectoparasitas em relação à contagem prévia à aplicação dos tratamentos constituídos por controle negativo (T1), alho picado e misturado ao concentrado a $100 \mathrm{~g}$ (T2) e $200 \mathrm{~g} \mathrm{vaca}^{-1} \mathrm{dia}^{-1}$ (T3) e por amitraz a $0,0025 \%$ (T4).Santa Maria, RS, 2007.

\begin{tabular}{|c|c|c|c|c|c|c|c|c|c|}
\hline \multirow{2}{*}{ Tratamentos } & \multicolumn{8}{|c|}{ Dias pós-tratamento } & \multirow{2}{*}{ Médias } \\
\hline & $1^{2}$ & $2^{2}$ & $3^{2}$ & $4^{2}$ & $5^{0}$ & $6^{6}$ & $7^{0}$ & $14^{a}$ & \\
\hline & \multicolumn{9}{|c|}{ Carrapatos (\%) } \\
\hline 1 & $140,84^{2}$ & $107,58^{2}$ & $197,48^{a}$ & $158,66^{n}$ & $170,38^{2}$ & $347,1^{a}$ & $129,69^{9}$ & $238,14^{2}$ & $186,24^{2}$ \\
\hline 2 & $92,50^{\circ}$ & $98,48^{3}$ & $110,64^{3}$ & $110,58^{3}$ & $94,51^{3}$ & $93,5^{3}$ & $112,36^{3}$ & $123,27^{3 \mathrm{~b}}$ & $100,47^{3}$ \\
\hline 3 & $88,73^{3}$ & $95,17^{3}$ & $102,70^{3}$ & $70,47^{3}$ & $53,62^{3}$ & $90,5^{3}$ & $84,92^{3}$ & $109,23^{3 t}$ & $90,93^{b}$ \\
\hline 4 & $16,88^{b}$ & $6,71^{\mathrm{b}}$ & $0,51^{b}$ & $0,00^{b}$ & $0,00^{b}$ & $0,8^{b}$ & $0,00^{\circ}$ & $22,28^{b}$ & $5.90^{\circ}$ \\
\hline \multirow[t]{2}{*}{$\mathrm{CV}(\%)$} & 21,74 & 15,63 & 19,68 & 15,34 & 15,60 & 37,55 & 13,66 & 21,68 & 21,32 \\
\hline & \multicolumn{9}{|c|}{ Mosca-dos-chifres (\%) } \\
\hline 1 & 99,13 & 85.22 & $94,05^{\mathrm{a}}$ & 95,49 & 130,68 & 120,79 & 227,3 & 73,10 & 115,72 \\
\hline 2 & 47,29 & 169,43 & $97,10^{\circ}$ & 189,76 & 79,29 & 158,19 & 282,8 & 132,38 & 144,53 \\
\hline 3 & 85,48 & 95,33 & $98,74^{\mathrm{a}}$ & 65,33 & 81,82 & 79,80 & 96,5 & 110,86 & 89,25 \\
\hline 4 & 92,45 & 119,05 & $24,18^{b}$ & 97,22 & 89,21 & 71,69 & 133,6 & 102.29 & 91,21 \\
\hline \multirow[t]{2}{*}{$C V(\%)$} & 34,52 & 45,65 & 24,41 & 31,74 & 31,28 & 28,18 & 25,84 & 16,57 & 30,44 \\
\hline & \multicolumn{9}{|c|}{ Mosca-dos-estábulos (+) mosca doméstica (\%) } \\
\hline 1 & 116,61 & $88,26^{3 b}$ & 59,51 & 118,69 & $75,73^{\text {st }}$ & 67,82 & 57,69 & 118,78 & $87,87^{3 \mathrm{~b}}$ \\
\hline 2 & 78,69 & $42,48^{b}$ & 42,48 & 38,24 & $18,75^{b}$ & 54,14 & 27,69 & 121,08 & $50,93^{-}$ \\
\hline 3 & 161,56 & $213,10^{\prime \prime}$ & 83,18 & 91,07 & $98,46^{\text {tb }}$ & 85,37 & 87,80 & 165.23 & $123,22^{\prime \prime}$ \\
\hline 4 & 68,52 & $31,93^{b}$ & 43,10 & 55,08 & $88,42^{3}$ & 40,83 & 129,81 & 52,62 & $63,79^{\mathrm{bc}}$ \\
\hline$C V(\%)$ & 16,80 & 19,98 & 60,76 & 17,22 & 20,23 & 12,92 & 20,21 & 13,89 & 23,48 \\
\hline
\end{tabular}

Médias com letras distintas, na coluna, indicam diferença significativa $(p<0,05)$ pelo teste de Duncan. CV = coeficiente de variação.

não se encaixem em recomendações que consideram um produto efetivo no controle desse parasita, superior a $90 \%$, havendo assim, necessidade da realização de novos estudos na tentativa de encontrar a dosagem segura de alho que possibilite um controle efetivo do parasita, já que em cães após administração de alho cru em pó ocorreram erosões nas mucosas estomacais (Hoshino et al., 2001).

Ressalta-se, nesta indicação, que os componentes estruturais e de extratos de plantas, por apresentarem composição química complexa, tendem apresentar lenta resistência parasitária (Chungsamarnyart \& Jiwajinda, 1992), diferentemente dos produtos químicos (Chagas et al., 2003).

Com relação ao controle de moscas, os resultados na Tabela 1 demonstram baixa eficácia dos produtos testados.

Observa-se que para a mosca-dos-chifres, os valores médios de infestação foram próximos ao valor médio de pré-tratamento, de 25 moscas vaca-1. Além da baixa eficácia dos produtos, verificou-se que houve grande variabilidade dos dados. Este resultado era esperado, considerando-se que o nível de infestação da mosca-dos-chifres é influenciado por fatores como coloração da pelagem e hormonais. A combinação destes e de outros fatores faz com que cada animal apresente maior ou menor susceptibilidade à infestação por esses dípteros (Bianchin \& Alves, 2002). Também Bianchin et al. (1999), avaliando o uso do alho em pó no sal mineralizado, não verificaram efeito no controle da mosca-dos-chifres.

Martins et al. (2002) alertaram para o problema da expansão da resistência da $H$. irritans aos produtos químicos e relataram que a eficácia da doramectina injetável, em estudo feito em Eldorado do Sul, RS, foi inferior a $50 \%$ no primeiro dia após o tratamento, com variação de 50,7 a $84,4 \%$ nos demais dias de observação, enquanto na Argentina (Rafaela, Santa Fé) a eficácia foi de $97,3 \%$ no primeiro dia e de 63,3 a 95,9 nos outros dias observados.

Para a mosca-dos-estábulos e para a mosca doméstica, o efeito dos produtos foi muito baixo. As diferenças encontradas $(p<0,05 \%)$ nos valores médios são atribuídas, possivelmente, às variações individuais dos animais, considerando-se que houve similaridade $(p>0,05 \%)$ entre os tratamentos constituídos pelo alho (200 $\mathrm{g} \mathrm{dia}^{-1}$ ) e o produto químico com a testemunha. Questiona-se, no entanto, o 
resultado observado no grupo em que se usou $100 \mathrm{~g}$ de alho, similar ao produto químico e superior aos demais tratamentos.

Quanto à análise sensorial do leite (Tabela 2), não foram detectadas diferenças nas características avaliadas de cor e sabor. Para o queijo, os atributos avaliados apresentaram diferenças $(p<0,05)$ entre as variáveis, provavelmente atribuídas à variabilidade entre animais e ao consumo de silagem, que em algum grau interfere nas características organolépticas do leite.

O coeficiente de variação da variável sabor residual, maior que o coeficiente das demais variáveis, deve-se, provavelmente, ao caráter relativo de sua avaliação (Dutcosky, 1996). Ressalta-se que, tanto no leite, quanto no queijo, não foram detectadas características de odor e sabor assemelhados aos do alho, em contraposição à grande quantidade ingerida pelos animais em pouco tempo. Acredita-se que estes resultados são devidos ao manejo alimentar utilizado, no qual o alho foi fornecido após a ordenha. Considerandose o intervalo mínimo entre ordenhas de 10h, houve a metabolização dos óleos voláteis, característicos ao sabor e odor do alho. Este odor detectado no

TABELA 2. Dados médios das variáveis de produção das vacas, análise sensorial do leite cru e do queijo tipo Minas-Frescal após à aplicação dos tratamentos constituídos por controle negativo (T1), alho picado e misturado ao concentrado a $100 \mathrm{~g}$ (T2) e $200 \mathrm{~g}_{\text {animal }} \mathrm{dia}^{-1}$ (T3) e por amitraz a 0,025\% (T4). Santa Maria, RS, 2007.

\begin{tabular}{|c|c|c|c|c|c|c|}
\hline \multirow{2}{*}{ Tratamentos } & \multicolumn{6}{|c|}{ Análise das variáveis de produçăo média das vacas } \\
\hline & \multicolumn{2}{|c|}{ Litros de leite dia $^{-1}$} & Peso & Idade & \multicolumn{2}{|c|}{ Estagio de lactaçăo } \\
\hline 1 & \multicolumn{2}{|l|}{17,8} & $592,25^{3}$ & 7,50 & \multicolumn{2}{|c|}{6,50} \\
\hline 2 & \multicolumn{2}{|c|}{15,20} & $498,50^{36}$ & 6,00 & \multicolumn{2}{|c|}{5,50} \\
\hline 3 & \multicolumn{2}{|c|}{14,55} & $548,75^{i 6}$ & $5, \infty 0$ & \multicolumn{2}{|c|}{7,75} \\
\hline 4 & \multicolumn{2}{|c|}{11,90} & $451,75^{\circ}$ & 4,25 & \multicolumn{2}{|c|}{6,25} \\
\hline $\mathrm{CV}(\%)$ & \multicolumn{2}{|l|}{35,5} & 11,79 & 36,51 & \multicolumn{2}{|c|}{41,54} \\
\hline \multirow{2}{*}{ Tratamentos } & \multicolumn{6}{|c|}{ Análise sensorial do leite cru (média) ${ }^{1}$} \\
\hline & \multicolumn{3}{|c|}{ Cor } & \multicolumn{3}{|c|}{ Odor } \\
\hline 1 & \multicolumn{3}{|c|}{4,53} & \multicolumn{3}{|c|}{$5,18^{a}$} \\
\hline 2 & \multicolumn{3}{|c|}{4,45} & \multicolumn{3}{|c|}{$4,58^{\mathrm{b}}$} \\
\hline 3 & \multicolumn{3}{|c|}{4,62} & \multicolumn{3}{|c|}{$4,93^{a b}$} \\
\hline 4 & \multicolumn{3}{|c|}{4,53} & \multicolumn{3}{|c|}{$4,83^{a b}$} \\
\hline $\mathrm{CV}(\%)$ & \multicolumn{3}{|c|}{8,09} & \multicolumn{3}{|c|}{16,48} \\
\hline \multirow{2}{*}{ Tratamentos } & & & e sensoria & do queijo & (media) $^{1}$ & \\
\hline & Aparência & Aroma & Cor & Sabor & Sabor residual & Textura \\
\hline 1 & $7,17^{c}$ & $5,90^{\circ}$ & $7,68^{b}$ & $6,21^{6}$ & $3,95^{\circ}$ & $5,90^{b}$ \\
\hline 2 & $7,37^{\mathrm{bc}}$ & $5,59^{\circ}$ & $8,32^{2}$ & $4,82^{d}$ & $4,83^{\circ}$ & $6,09^{b}$ \\
\hline 3 & $7,93^{8}$ & $7,53^{5}$ & $8,28^{3}$ & $7,03^{b}$ & $4,45^{(6)}$ & $7,72^{3}$ \\
\hline 4 & $7,72^{\omega b}$ & $8,35^{\circ}$ & $8,56^{\circ}$ & $7,84^{\circ}$ & $3,20^{\circ}$ & $7,51^{a}$ \\
\hline $\mathrm{CV}(\%)$ & 11,22 & 20,21 & 13,14 & 21,63 & 32,31 & 22,55 \\
\hline
\end{tabular}

Médias com letras distintas, na coluna, indicam diferença significativa $(p<0,05)$ pelo teste de Duncan. ${ }^{1}$ Dados médios referentes às analises efetuadas nos $1^{\circ}, 2^{\circ} \underline{0}$ e $3^{\circ} \underline{0}$ dias após a aplicação dos tratamentos. $C V=$ coeficiente de variação.

ar expirado ou no leite pode ser detectado, notadamente entre uma e duas horas após o consumo de alho (Glazier, 1960).

Assim, conclui-se que o amitraz mostrouse efetivo no controle do carrapato. Os tratamentos constituídos por diferentes níveis de alho apresentaram efeito parcial no controle do carrapato. Tanto o produto químico quanto os fitoterápicos não apresentaram efeito nas moscas-dos-chifres e mosca dos estábulos (+) mosca doméstica. Os produtos utilizados não influenciaram nas propriedades organolépticas do leite e do queijo tipo Minas- Frescal. 


\section{REFERÊNCIA}

ALVARENGA, L.C. et al. Alteração da carga de carrapatos de bovinos sob a ingestão de diferentes níveis do resíduo do beneficiamento do alho. Ciência Agrotécnica, v.28, n.4, p.906-12, 2004.

ANDREOTTI, R. Imunoproteção de bovinos contra o carrapato Boophilus microplus a partir de inibidores de serinoproteases. Revista Brasileira de Parasitologia Veterinária, v.13, supl.1, p.140-5, 2004.

ANKRI, S.; MIRELMAN, D. Antimicrobial properties of allicin from garlic. Microbes and Infection, v.1, n.2, p.1259, 1999.

BIANCHIN, I.; ALVES, R.G.O. Mosca-dos-chifres, Haematobia irritans: comportamento e danos em vacas e bezerros Nelore antes da desmama. Pesquisa Veterinária Brasileira, v.22, n.3, p.109-113, 2002.

BIANCHIN, I. et al. Eficiência do pó de alho (Allium sativum L.) no controle dos parasitas de bovinos. Campo Grande: Embrapa Gado de Corte, 1999. 31p. (Boletim de Pesquisa, 8).

CHAGAS, A.C.S. et al. Sensibilidade do carrapato Boophilus microplus a solventes. Ciência Rural, v.33, n.1, p.109-14, 2003.

CHUNGSAMARNYART, N.; JIWAJINDA, S. Acaricidal activity of volatile oil from lemon and citronella grasses on tropical cattle ticks. Kasetsart Journal, v.26, n.5, p.4651, 1992.

DUTCOSKY, S.D. Análise sensorial de alimentos. Curitiba: Champagnat, 1996. 123p.

FARIA, N.M.X. et al. Intoxicação por agrotóxicos no Brasil: os sistemas oficiais de informação e desafios para realização de estudos epidemiológicos. Ciência e Saúde Coletiva, v.12, n.1, p. 2007.

FURLONG, J. Controle estratégico do carrapato dos bovinos. A Hora Veterinária, v.23, n.137, p.53-6, 2004.

GLAZIER, Z.R. Milk Flavor Improvement. Connecticut: Connecticut Agricultural Extension, 1960. 10p. (Boletim técnico, 10).

GRISI, L. et al. Impacto econômico das principais ectoparasitoses em bovinos no Brasil. A Hora Veterinária, v.21, n.125, p.8-10, 2002.

HOSHINO, T.; KASHIMOTO, N.; KASUGA, S. Effects of
Garlic Preparations on the Gastrointestinal Mucosa. The Journal of Nutrition, v.131, Supl.1, p.1109S-13S, 2001. HEIMERDINGER, A. et al. Extrato alcoólico de capimcidreira no controle do Boophilus microplus em bovinos. Revista Brasileira de Parasitologia Veterinária, v.15, n.1, p.37-9, 2006.

JONSSON, N. Integrated control programs for ticks on dairy cattle: an examination of some possible components. Queensland: FAO, 2006. 63p.

KYO, E. et al. Immunomodulatory Effects of Aged Garlic Extract. The journal of nutrition, v.131, supl.1, p.1075S9S, 2001.

LAWLESS, H.T.; HEYMANN, H. Sensory evaluation of food: principles and practices. New York: Chapman \& Hall. 1998. 819p.

MARTINS, R.J. et al. Ação da doramectina injetável em Haemaobia irritans em bovinos naturalmente infestados: Resultados de observações espontâneas no Brasil e Argentina. Ciência Rural, v.34, n.4, p.633-6, 2002.

MOLENTO, M.B. et al. Método Famacha como parâmetro clínico individual de infecção por Haemonchus contortus em pequenos ruminantes. Ciência Rural, v.34, n.4, p.1139-45, 2004.

OLIVEIRA, A.A.; AZEVEDO, H.C. Resistência do carrapato Boophilus microplus a carrapaticidas em bovinos de leite na região dos tabuleiros costeiros de Sergipe. Científica Rural, v.7, n.2, p.64-71, 2002.

ROEL, A.R. Utilização de plantas com propriedades inseticidas: uma contribuição para o Desenvolvimento Rural Sustentável. Revista Internacional de Desenvolvimento Local, v.1, n.2, p.43-50, 2002.

SAS INSTITUTE, SAS User's guide: statistics, Version 6.11. Cary: SAS Institute, 1997. 1167p.

URBINA, J.A. et al. Inhibition of phosphatidylcholine biosynthesis and cell proliferation in Trypanosoma cruzi by ajoene, an antiplatelet compound isolated from garlic. Instituto Venezolano de Investigaciones Cientificas, v.45, n.12, p.2381-7, 1993.

VIEIRA, L.S.; CAVALCANTE, A.C.R. Resistência antihelmíntica em rebanhos caprinos no Estado do Ceará. Pesquisa Veterinária Brasileira, v.19, n.3-4, p.99-103, 1999. 